This item was submitted to Loughborough's Research Repository by the author.

Items in Figshare are protected by copyright, with all rights reserved, unless otherwise indicated.

\title{
Motion-resonant modes of large articulated damped oscillators in waves
}

PLEASE CITE THE PUBLISHED VERSION

http://dx.doi.org/10.1016/j.jluidstructs.2014.06.012

\section{PUBLISHER}

(c) Elsevier Ltd

\section{VERSION}

AM (Accepted Manuscript)

\section{PUBLISHER STATEMENT}

This work is made available according to the conditions of the Creative Commons Attribution-NonCommercialNoDerivatives 4.0 International (CC BY-NC-ND 4.0) licence. Full details of this licence are available at: https://creativecommons.org/licenses/by-nc-nd/4.0/

\section{LICENCE}

CC BY-NC-ND 4.0

\section{REPOSITORY RECORD}

Renzi, Emiliano, and F. Dias. 2019. "Motion-resonant Modes of Large Articulated Damped Oscillators in Waves". figshare. https://hdl.handle.net/2134/17134. 


\title{
Motion-Resonant Modes of Large Articulated Damped Oscillators in Waves
}

\author{
E. Renzi ${ }^{\mathrm{a}, *}$, F. Dias ${ }^{\mathrm{a}, \mathrm{b}}$ \\ ${ }^{a}$ UCD School of Mathematical Sciences, University College Dublin, Belfield Dublin 4, \\ Ireland \\ ${ }^{b}$ Centre de Mathématiques et de Leurs Applications (CMLA), Ecole Normale Supérieure \\ de Cachan, 94235 Cachan, France
}

\begin{abstract}
Using a semi-analytical approach, we show that an articulated system of large damped oscillators in the open ocean can be resonated by incoming waves at multiple frequencies. As an application, energy extraction from the system is modelled when the oscillators are used as flap-type wave energy converters. A new parameter - the absorption efficiency - is introduced to analyse the performance of the system at resonance. This allows us to identify the occurrence of detrimental processes near the resonant frequencies, which reduce the sustainability of the energy conversion process. This result challenges the diffused belief that large flap-type wave energy converters must be designed to resonate, which is based on the use of inappropriate performance descriptors.
\end{abstract}

Keywords: Fluid-structure interaction, multiple resonance, wave energy

\section{Introduction}

In this paper we analyse the resonant wave interaction with large articulated moving structures. We believe that this topic has received too little attention in the past, despite its importance in several engineering applications, like e.g. wave energy conversion. Indeed, many studies have been carried out to investigate the occurrence of resonant amplifications resulting

\footnotetext{
*Corresponding author

Email addresses: emiliano.renzi@ucd.ie (E. Renzi), frederic.dias@ucd.ie (F. Dias)
} 
from wave-structure interactions in acoustics (see for example Farhat et al., 2010), optics (see for example Ruan and Fan, 2010) and hydrodynamics (Budal, 1977; Falnes, 1980; Thomas and Evans, 1981; Heathershaw, 1982; Mei et al., 1988, 1994; Maniar and Newman, 1997; Sammarco et al., 1997a,b; Porter and Evans, 1999; Hu and Chan, 2005; Adamo and Mei, 2005; Sammarco and Renzi, 2008; Renzi and Sammarco, 2010; Stefanakis et al., 2011; Hu et al., 2011; Renzi and Dias, 2012, 2013a,b; Renzi et al., 2014a,b). In the latter discipline, a significant effort was directed towards understanding the mechanisms of resonant amplification of water waves impinging on fixed structures, such as periodic bathymetric formations or surface scatterers. Notable results of such endeavour were the discovery of the Bragg scattering of ocean surface waves by a periodic bathymetry (Heathershaw, 1982; Mei et al., 1988) and the identification of resonant long-wave modes on a beach (Sammarco and Renzi, 2008; Renzi and Sammarco, 2010; Stefanakis et al., 2011). Interaction of water waves with fixed structures is a living matter also in offshore ocean engineering. In this field, a significant advancement of knowledge has been achieved with the discovery of the Rayleigh-Bloch surface waves trapped along arrays of cylinders (Maniar and Newman, 1997; Porter and Evans, 1999). Concerning the interaction of water waves with moving bodies, the theory of interacting point oscillators in water waves is indeed a subject with honourable history (Budal, 1977; Falnes, 1980; Thomas and Evans, 1981). Such theory is based on the assumption that the horizontal extension $w$ of each oscillator is much smaller than the wavelength $\lambda$, i.e. $w / \lambda \ll 1$. This hypothesis allows one to neglect the scattering of the incident waves on the bodies, hence reducing the complexity of the mathematical formulation associated with the problem. Despite such advancements, less effort has been paid towards the study of resonant water wave interactions with large articulated moving structures, like for example bottom-hinged rectangular flaps, whose dimensions are not much smaller than the wavelength: $w / \lambda \lesssim 1$. Such bodies induce substantial changes in the pattern of wave propagation (Mei et al., 2005). As a consequence, their interplay with incident waves can generate resonant interactions which cannot be explained with the traditional point-oscillator theory. Mei et al. (1994) and Sammarco et al. (1997a) showed for the first time that incident waves can trigger largeamplitude pitching motions of an array of narrowly spaced, large rectangular gates in a channel. Note that the gates of Mei et al. (1994) and Sammarco et al. (1997a) move in the absence of any power take-off damping. Lately, a resonant mechanism has been shown to occur for the same oscillators when 
located at the intersection of a straight channel and a semi-infinite domain (Adamo and Mei, 2005) and for multiple arrays of such oscillators in a channel (Sammarco et al., 2014). However, the existence of resonant modes for an articulated system of large, externally damped oscillators in the open ocean has not yet been investigated. Here we shall undertake this investigation by considering a system of large rectangular oscillators, hinged upon a bottom foundation and damped by a resistive action proportional to their angular velocity. Such system has an important application in the field of wave energy extraction, where these oscillators are known as "flap-type absorbers" (Renzi et al., 2014b). The flap-type absorber represents the reference mathematical model used for the design of several wave energy converters (WECs), like for example the Oyster ${ }^{\circledR}$ Oscillating Wave Surge Converter (OWSC, see Whittaker and Folley, 2012; Renzi and Dias, 2012, 2013a,b; Renzi et al., 2014a) manufactured by Aquamarine Power, with a maximum generating capacity of $800 \mathrm{~kW}$ (www.aquamarinepower.com).

This paper has the following aims:

(i) To model the fluid-structure interaction for a system of $M$ oscillators (Section 2).

(ii) To introduce the concept of motion resonance, which describes the resonant phenomenon triggered by the oscillators moving as an articulated system of interdependent bodies (Section 3).

(iii) To investigate the influence of motion-resonant modes on the efficiency of the oscillators as wave energy converters. A new parameter - the absorption efficiency - is introduced to undertake a qualitative analysis of the system at resonance. Such analysis would be otherwise inaccurate if conducted with existing performance parameters (Section $3)$.

An application is made for the case of three in-line oscillators, as a fundamental cluster for larger wave farms of multiple converters. It is shown that the excitation of body-to-body resonance is detrimental to the efficiency of wave energy absorption processes (Section 4). 

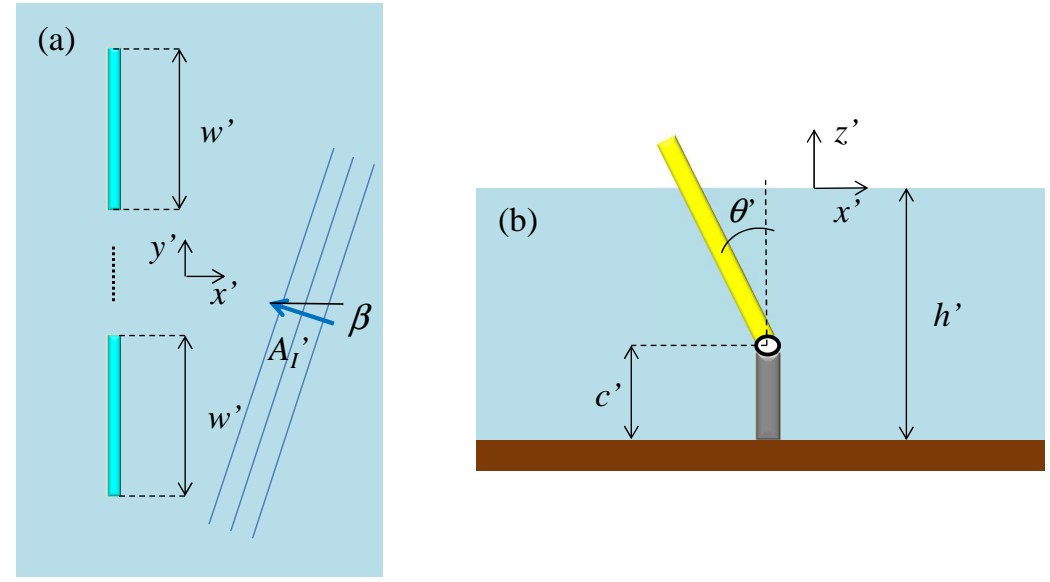

Figure 1: Geometry of the system. (a) plan view, (b) section.

\section{Model}

\subsection{Governing equations}

Within the framework of the potential flow theory in water of intermediate depth (Budal, 1977; Falnes, 2002; Mei et al., 2005), consider inviscid and irrotational water waves in a fluid domain of depth $h^{\prime}$ unbounded in the horizontal $\left(x^{\prime}, y^{\prime}\right)$ plane. Primes indicate physical dimensional variables. The domain is pierced by a finite number $M$ of identical flap-type oscillators, i.e. externally damped flaps of rectangular shape, shown in Fig. 1. The oscillators are aligned along the $y^{\prime}$ direction. The axis $z^{\prime}$ rises up from the unperturbed water level $z^{\prime}=0$. As mentioned in the Introduction, the flap-type absorber is the mathematical reference model for the OWSC, a large buoyant flap used for wave energy extraction in the nearshore environment (Renzi and Dias, 2012, 2013a,b; Renzi et al., 2014a). Only in-line configurations will be investigated in this paper, as loss of symmetry in small arrays of OWSCs is likely to be accompanied by a reduction in efficiency (Renzi et al., 2014a; Sarkar et al., 2014). The oscillators are set into motion by incident waves of amplitude $A_{I}^{\prime}$, period $T^{\prime}$ and angular frequency $\omega^{\prime}=2 \pi / T^{\prime}$ incoming along a direction which forms an angle $\beta$ with the $x^{\prime}$ axis. Each of the oscillators is represented as a large buoyant flap of width $w^{\prime}$ hinged on a base of height $c^{\prime}$ at the bottom of the fluid domain, moving under the action of the incoming wave field with one degree of freedom, i.e. the pitching angle $\theta^{\prime}{ }_{m}\left(t^{\prime}\right), m=1,2, \ldots, M ; t^{\prime}$ denotes time. It is assumed that the amplitude 
of the wave field is small with respect to the width of each device, as it happens in reality for most of the operational life of the system (Gallagher et al., 2013). Hence following the linearised theory of Renzi et al. (2014a) and Sarkar et al. (2014), the velocity potential $\Phi^{\prime}\left(x^{\prime}, y^{\prime}, z^{\prime}, t^{\prime}\right)$ must satisfy the Laplace equation

$$
\nabla^{\prime 2} \Phi^{\prime}=0
$$

in the fluid domain, where $\nabla^{\prime} f=\left(f_{x^{\prime}}, f_{y^{\prime}}, f_{z^{\prime}}\right)$ and subscripts denote differentiation with respect to the relevant variable. On the free-surface, the kinematic-dynamic boundary condition reads

$$
\Phi_{t^{\prime} t^{\prime}}^{\prime}+g \Phi_{z^{\prime}}^{\prime}=0, \quad z^{\prime}=0
$$

where $g$ is the acceleration due to gravity, while at the bottom the no-flux condition requires

$$
\Phi_{z^{\prime}}^{\prime}=0, \quad z^{\prime}=-h^{\prime}
$$

Finally, the kinematic condition on the surface of the oscillators writes

$$
\Phi_{x^{\prime}}^{\prime}=-\theta_{m, t^{\prime}}^{\prime}\left(t^{\prime}\right)\left(z^{\prime}+h^{\prime}-c^{\prime}\right) H\left(z^{\prime}+h^{\prime}-c^{\prime}\right)
$$

on each element $m=1, \ldots, M$, where the Heaviside step function assures absence of flux through the foundation. Following Adamo and Mei (2005), Renzi and Dias (2012, 2013a,b), Renzi et al. (2014a) and Sarkar et al. (2014), in Eq. (4) the flap thickness is considered immaterial for the calculation of the potential. The boundary-value problem (1)-(4) has been already studied and solved by Renzi et al. (2014a) for an array of flap-type absorbers by employing the Green integral theorem and a series expansion in terms of the Chebyshev polynomials. However, Renzi et al. (2014a) did not investigate the system motion-resonant modes, leaving such analysis to further work which is undertaken in this paper. In the following, we shall briefly summarise the method of Renzi et al. (2014a) and eventually delve into the investigation of the motion-resonant modes of the system. Let us introduce the nondimensional variables

$$
(x, y, z)=\left(x^{\prime}, y^{\prime}, z^{\prime}\right) / w^{\prime}, \quad t=\sqrt{g / w^{\prime}} t^{\prime}, \quad \epsilon \theta_{m}=\theta_{m}^{\prime}
$$

and constants

$$
(h, c)=\left(h^{\prime}, c^{\prime}\right) / w^{\prime}, \quad A_{I}=A_{I}^{\prime} / A^{\prime}
$$


where $A^{\prime}$ is the amplitude scale of the incident wave and $\epsilon=A^{\prime} / w^{\prime} \ll 1$ is the physical scale of the oscillations. Let us further decompose the nondimensional potential $\Phi$ and the amplitude of rotation of the $m$ th oscillator $\theta_{m}$ as

$$
\Phi(x, y, z, t)=\Re\left\{\left(\phi_{I}+\phi_{D}+\sum_{m=1}^{M} \Omega_{m} \phi_{R}^{(m)}\right) \mathrm{e}^{-\mathrm{i} \omega t}\right\} ; \quad \theta_{m}=\Re\left\{\Theta_{m} \mathrm{e}^{-\mathrm{i} \omega t}\right\},
$$

respectively. In the latter,

$$
\phi_{I}(x, y, z)=-\frac{\mathrm{i} A_{I}}{\omega} \frac{\cosh k(z+h)}{\cosh k h} \mathrm{e}^{-\mathrm{i} k x \cos \beta+\mathrm{i} k y \sin \beta}
$$

is the spatial component of the incident wave potential, where $k$ solves the dispersion relation $\omega^{2}=k \tanh k h$. $\phi_{D}$ is the diffraction potential and $\phi_{R}^{(m)}$ is the radiation potential emanating by the $m$ th oscillator when all the other oscillators are at rest. $\Omega_{m}=\mathrm{i} \omega \Theta_{m}$ and $\Theta_{m}$ is the complex angular rotation of the $m$ th flap. Finally, it is required that $\phi_{D}$ and $\phi_{R}^{(m)}$ are outgoing perturbations in the far field. Renzi et al. (2014a) and Sarkar et al. (2014) found semi-analytical expressions for the diffraction potential $\phi_{D}$ and each radiation potential $\phi_{R}^{(m)}$ in Eq. (7) by applying the Green integral theorem in the fluid domain, thus obtaining a system of hypersingular integral equations in terms of the jump in potentials across the flaps (see Appendix A of Sarkar et al., 2014). Such equations are appropriately de-singularised by employing a series expansion in term of the Chebyshev polynomials of the second kind. The final results of this procedure, i.e. the mathematical expressions of $\phi_{D}$ and $\phi_{R}^{(m)}$, are detailed in Sarkar et al. (2014), which the interested reader is referred to.

\subsection{Equation of motion}

The full solution of the boundary value problem (1)-(4) still requires to find the unknown amplitudes of rotation $\Theta_{m}$ in Eq. (7) and Eq. (4). This task can be accomplished by solving the following system of equations of motion for the oscillators in the frequency domain (Falnes, 2002; Mei et al., 2005; Renzi et al., 2014a; Sarkar et al., 2014):

$$
\left[-\omega^{2}(\mathbf{I}+\mathbf{M}(\omega))+\mathbf{C}-\mathrm{i} \omega(\mathbf{N}(\omega)+\mathbf{H}(\omega))\right]^{\mathrm{T}} \boldsymbol{\Theta}(\omega)=\mathbf{F}(\omega) .
$$

The latter represents the dynamic equilibrium between inertia, buoyancy, external damping and hydrodynamic torques acting on each oscillator. In 
Eq. (8), the terms $\mathbf{I}, \mathbf{C}$ and $\mathbf{H}(\omega)$ are diagonal $M \times M$ matrices. Their $(m, m)$ entries, $I_{m}, C_{m}$ and $\eta_{m}(\omega)$ respectively, are the second moment of inertia, the restoring buoyancy torque and the external damping rate of each oscillator. The external damping rate can represent, for example, the damping action of power take-off mechanisms (e.g. generators) when the oscillators are used as wave energy converters. Again in Eq. (8), $\mathbf{M}(\omega)$ and $\mathbf{N}(\omega)$ are, respectively, the added-inertia and the radiation damping matrices. The radiation damping matrix $\mathbf{N}(\omega)$ is also termed radiation resistance matrix in general physics. The sum $\mathbf{Z}(\omega)=\mathbf{N}(\omega)+i \omega \mathbf{M}(\omega)$ corresponds to the radiation impedance matrix in acoustics and electromagnetics (Falnes, 2002). The scalar components $\mu_{\alpha \beta}(\omega)$ of $\mathbf{M}(\omega)$ and $\nu_{\alpha \beta}(\omega)$ of $\mathbf{N}(\omega)$ result from the hydrodynamic actions on body $\beta$ when body $\alpha$ is moving and enjoy the symmetry property $\mu_{\alpha \beta}=\mu_{\beta \alpha}, \nu_{\alpha \beta}=\nu_{\beta \alpha}$ (Falnes, 1980; Mei et al., 2005; Renzi et al., 2014a). Still in Eq. (8), $\boldsymbol{\Theta}(\omega)$ is a vector whose $m$ th scalar component is the sought complex amplitude of oscillations $\Theta_{m}(\omega)$. Finally, $\mathbf{F}(\omega)$ is a vector whose $m$ th scalar term is the complex exciting torque $F_{m}(\omega)$ acting on body $m$ when all bodies are held fixed in incoming waves (Falnes, 1980; Mei et al., 2005; Renzi et al., 2014a). In this paper, the matrices I, C and $\mathbf{H}(\omega)$ in Eq. (8) are assumed to be given. The added-inertia matrix $\mathbf{M}(\omega)$ and the radiation damping matrix $\mathbf{N}(\omega)$ are obtained by integrating the spatial components of the radiation potentials $\phi_{R}^{(m)}$ on the surface of each flap, as shown in Renzi et al. (2014a). This yields semi-analytical expressions which are then evaluated numerically. Finally, the exciting torque vector $\mathbf{F}$ is evaluated by integrating the spatial component of the diffraction potential $\phi_{D}$ on the surface of each flap. Again, this yields semi-analytical expressions which can be evaluated numerically at very low computational cost. The computational aspects of the numerical evaluation of the terms in Eq. (8) are detailed in Renzi et al. (2014a) and Sarkar et al. (2014).

The mathematical model summarised in this section has significant practical importance in applications to wave energy systems. Indeed Eqs (1)-(8) constitute the mathematical model termed "flap-type absorber" by Renzi et al. (2014b), which is at the basis of the OWSC technology. In the literature, the flap-type absorber theory has been extensively validated against numerical and experimental data for several layouts of practical interest. Renzi and Dias (2012) first validated the model for a single flap in a channel with respect to numerical data obtained at Queen's University Belfast (QUB, U.K.) with the commercial software WAMIT, for monochromatic in- 
cident waves. Renzi and Dias (2012) also showed good agreement between the flap-type absorber results and the wave tank data obtained at QUB during an experimental campaign on the Oyster WEC, again in monochromatic waves. Renzi and Dias (2013a) validated their model of an infinite array of flap-type absorbers in the open ocean against numerical results obtained with a finite-element numerical model developed by the University of Roma Tre (Italy). Later, Sarkar et al. (2013) successfully compared the flap-type absorber results in random seas with wave-tank data obtained at QUB. Finally, Renzi et al. (2014a) showed very good agreement between the semi-analytical model of an in-line array of flap-type absorbers in monochromatic seas and numerical data obtained with a finite-element model. The theoretical model summarised in this section is hence fully validated regarding its computational coherence and can be successfully employed to simulate the behaviour of actual OWSC systems.

Despite such intensive analysis on the flap-type absorber, none of the aforementioned authors have investigated the mechanism of multiple resonance for this system in the open ocean, which is analysed in the next section.

\section{Motion Resonance}

\subsection{Natural modes}

As is well known in the theory of dynamical systems, the natural modes of a system of oscillators are the basic configurations of the free undamped motion (Kreyszig, 2006). Mathematically, we seek the solutions of the system of equations of motion (8) when the damping terms $\mathbf{N}$ and $\mathbf{H}$ and the exciting torque $\mathbf{F}$ are set to zero:

$$
\left[-\omega^{2}(\mathbf{I}+\mathbf{M}(\omega))+\mathbf{C}\right]^{T} \boldsymbol{\Theta}(\omega)=\mathbf{0} .
$$

If all the oscillators are similar, e.g. they have identical moment of inertia $I$ and buoyancy torque $C$, then (9) can be conveniently re-written as

$$
\left[C^{-1}(\mathbf{I}+\mathbf{M}(\omega))-\alpha(\omega) \mathbb{I}_{M}\right] \mathbf{\Theta}(\omega)=\mathbf{0}, \quad \omega>0,
$$

where $\alpha(\omega)=1 / \omega^{2}, \mathbb{I}_{M}$ is the $M \times M$ identity matrix and the symmetry property of $\mathbf{M}$ has been used. Eq. (10) is an implicit eigenvalue problem for $\omega$. The eigenvalues are determined by solving the characteristic equation

$$
\Delta(\omega)=\operatorname{det}\left[C^{-1}(\mathbf{I}+\mathbf{M}(\omega))-\alpha(\omega) \mathbb{I}_{M}\right]=0, \quad \omega>0,
$$


which is an implicit non-linear equation in $\omega$, admitting $N$ different real solutions $\omega_{i}, i=1, \ldots, N$, with $N \leq M$. Note that, for given $I$ and $C$, Eq. (11) depends only on the added-inertia matrix $\mathbf{M}$. The eigenvalues $\omega_{i}$ solution of Eq. (11) are hence a radiative property of the system and do not depend on the incident wave characteristics. This is coherent with the position $\mathbf{F}=\mathbf{0}$ made to obtain Eqs (9)-(11). Physically, the $\omega_{i}$ represent the natural frequencies of the system (Kreyszig, 2006). Once those are known, the relevant natural modes are found by setting $\Theta_{1}=1$ in Eq. (9) and then solving for the remaining components of the vector $\Theta$ with $\omega=\omega_{i}$ in succession (for a similar procedure see also Sammarco et al., 2014). Computational aspects related to the numerical solution of the implicit equation (11) are detailed in Appendix A.

In the presence of damping (external plus radiation) and incident waves, the system resonates when the incident wave frequency is close to any of the natural frequencies, i.e. $\omega \simeq \omega_{i}$ (Kreyszig, 2006). Such phenomenon occurs because the oscillators move as an articulated system of interdependent bodies and is therefore termed motion resonance. Note that this type of resonance, due to body-to-body interactions, is intrinsically different from the resonance of a single floating body by buoyancy alone (Mei et al., 2005; Adamo and Mei, 2005), in which case the resonant frequency satisfies the well-known implicit relation

$$
\omega_{0}=\sqrt{\frac{C}{I+\mu_{11}\left(\omega_{0}\right)}} .
$$

In the following sections we shall investigate the effects of motion resonance on the behaviour of the system, when the oscillators are used to extract energy from the waves.

\subsection{Effect on wave power extraction}

Resonance of damped forced oscillators manifests with the excitation of large oscillations (Kreyszig, 2006). When the oscillators are used to extract energy from the sea, this mechanism suggests that more power is captured by the system if it is set to resonate by the incident waves. Indeed the average power captured by a system of $M$ identical flap-type absorbers oscillating at a frequency $\omega$ during a period $T=2 \pi / \omega$ is given by

$$
P(\omega)=\frac{\omega^{2}}{2} \eta(\omega) \sum_{m=1}^{M}\left|\Theta_{m}(\omega)\right|^{2},
$$


where the PTO damping $\eta$ is common to all the absorbers (Babarit, 2010; Renzi et al., 2014a). Note that in Eq. (13) the $\Theta_{m}$ 's also depend on the PTO damping $\eta$ via the system (8). Hence $P(13)$ is a non-linear function of $\eta(\omega)$, which in turn depends on the PTO strategy adopted by the manufacturer. Here, for each given frequency $\omega$, we shall use the value of $\eta(\omega)$ which optimises the absorbed power $P(\omega)$ in Eq. (13). The optimisation is performed numerically as outlined in Renzi et al. (2014a). Clearly, for given non-zero $\eta(\omega)$, peaks of the $\left|\Theta_{m}\right|$ expected at resonant frequencies (Kreyszig, 2006) determine the absorbed power $P(13)$ to peak at resonance as well. However, large values of $\left|\Theta_{m}\right|$ violate the small-amplitude oscillation assumption, resulting in unrealistic large values for the absorbed power $P(13)$ at resonance. Nevertheless, an insight on the system resonant behaviour can still be obtained with the linearised theory by using the following argument. When the incident waves encounter the oscillators, part of the incident power is indeed absorbed by the external damping mechanism. However, part of the incident wave power is transmitted back to the ocean, associated with the rate of work done by the oscillating bodies to the fluid (Adamo and Mei, 2005; Mei et al., 2005):

$$
\begin{aligned}
W(\omega) & =\sum_{\alpha=1}^{M} \sum_{\beta=1}^{M} \nu_{\alpha \beta} \overline{\theta_{\alpha, t} \theta_{\beta, t}} \\
& =\frac{\omega^{2}}{2} \sum_{\alpha=1}^{M} \sum_{\beta=1}^{M} \nu_{\alpha \beta}(\omega)\left|\Theta_{\alpha}(\omega)\right|\left|\Theta_{\beta}(\omega)\right| \cos \left(\delta_{\alpha}(\omega)-\delta_{\beta}(\omega)\right) .
\end{aligned}
$$

In the latter, the bar indicates the average over a period and $\delta_{m}=\arg \left(\Theta_{m}\right) \in$ $(-\pi, \pi]$. The absorption process taking place in the system can be analysed by defining the absorption efficiency

$$
\begin{aligned}
Q_{M}(\omega) & =\frac{P(\omega)}{W(\omega)+P(\omega)} \\
& =\left(1+\frac{\sum_{\alpha=1}^{M} \sum_{\beta=1}^{M} \nu_{\alpha \beta}(\omega)\left|\Theta_{\alpha}(\omega)\right|\left|\Theta_{\beta}(\omega)\right| \cos \left(\delta_{\alpha}(\omega)-\delta_{\beta}(\omega)\right)}{\eta(\omega) \sum_{\beta=1}^{M}\left|\Theta_{\beta}(\omega)\right|^{2}}\right)^{-1},
\end{aligned}
$$

as the ratio between the absorbed power and the total output power (lost + absorbed). A good design must try not only to enhance the power capture $P$ 
(13), but also to reduce the lost power $W$, thus increasing $Q_{M}$ (15) towards its theoretical unit upper limit. Note that the absorption efficiency (15) is different from the capture factor used in previous work (Renzi and Dias, 2012, 2013a,b) to assess the performance of WEC systems in small-amplitude oscillations. The capture factor is defined as the ratio between the absorbed power $P$ and the incident wave power $P_{\text {inc }}$ on the system:

$$
C_{F}(\omega)=\frac{P(\omega)}{P_{\text {inc }}(\omega)}=\frac{\omega^{2} \eta(\omega)}{2 P_{\text {inc }}(\omega)} \sum_{m=1}^{M}\left|\Theta_{m}(\omega)\right|^{2} .
$$

Naturally, for a given frequency, the capture factor (16) is maximum when the absorbed power is maximised (Falnes, 2002; Mei et al., 2005; Cruz, 2008). However, using the capture factor (16) to assess the behaviour of the system at resonance has one major drawback. As already mentioned, the linear theory would predict exceedingly large values of the $\Theta_{m}$ 's at resonance, so that the capture factor (16) would be overestimated too, rising well above unity (Folley et al., 2007). For this reason, within the framework of the linearised theory, the capture factor (16) is as inappropriate as $P$ (13) to assess the performance of the system at motion resonance. On the contrary, note that the absorption efficiency defined in Eq. (15) depends on the relative amplitudes of rotation of the absorbers (see also the examples in the following), which are not affected by the resonant growth of the single $\Theta_{m}$ 's, as shown in the following Section 4. This allows us to obtain realistic values of $Q_{M}$ also at resonance, thus permitting an extension of the linear theory to investigate motion-resonant cases. Clearly, Eq. (15) only allows one to perform a qualitative analysis of the system at motion resonance, whose quantitative aspects should be examined further with an accurate numerical simulation, e.g. with bespoke computational fluid dynamics (CFD) codes. The latter can be designed to account for large oscillations and to reproduce the associated dissipative vortex dynamics in detail (Rafiee and Dias, 2013; Wei et al., 2013a,b; Bouscasse et al., 2013; Liu et al., 2014). Nevertheless, Eq. (15) allows an initial assessment of the system resonant behaviour to be made at much lower computational costs than CFD and as such is worth investigating.

For example, in the case of one absorber, Eq. (15) provides very useful analytical means to assess the effect of body resonance on the performance 
of the device. For $M=1$ Eq. (15) simplifies as follows:

$$
Q_{1}=\left(1+\frac{\nu_{11}(\omega)}{\eta(\omega)}\right)^{-1}
$$

which, for given $\eta(\omega)$, is independent of the angle of rotation of the flap. Now, for a single absorber the expression of the PTO damping which optimises $P$ (13) is well-known to be (Falnes, 2002; Renzi and Dias, 2013b):

$$
\eta(\omega)=\sqrt{\frac{\left[C-\left(I+\mu_{11}(\omega)\right) \omega^{2}\right]^{2}}{\omega^{2}}+\nu_{11}(\omega) .}
$$

Since $\eta(\omega) \geq \nu_{11}(\omega)$, then Eq. (17) yields $Q_{1} \geq 1 / 2$ for a single absorber. Furthermore, in the special case $\omega=\omega_{0}$ (i.e. at body resonance, see Eq. (12)), Eq. (17) together with (18) and (12) yield the minimum absorption efficiency

$$
Q_{1}\left(\omega_{0}\right)=1 / 2 \text {. }
$$

The latter expression physically means that, if tuned to resonance, a single oscillator in the open ocean can absorb at most half of the total output power, the other half being reintroduced in the fluid by its motion. This happens since the larger amplitude of rotation at resonance increases not only the power capture, but also the radiated power, which is an unwanted loss.

For multiple bodies $(M>1)$, Eq. (15) cannot be investigated analytically and semi-analytical evaluation is necessary. In this case, the behaviour of $Q_{M}(\omega)$ depends on the layout of the system, so that general conclusions on the effect of motion resonance on the absorption efficiency cannot be made a priori. In the following section we shall consider the case study of three in-line flap-type absorbers under normally incident waves, which constitute the basic cluster for more complex array developments. We shall show that motion resonance is accompanied by a substantial loss of power in the ocean.

\section{Numerical application}

In this section we present a numerical application of the theoretical results of Section 2 and Section 3, considering a cluster of three in-line large damped oscillators of the flap-absorber type. The geometry of the flaps is reported in table 1 . The spacing between the flaps is $s^{\prime}=10 \mathrm{~m}$, the waves are normally incident on the system. Each oscillator resembles the geometry 


\begin{tabular}{ccc}
\hline Flap width $(\mathrm{m})$ & Foundation height $(\mathrm{m})$ & Water depth $(\mathrm{m})$ \\
$w^{\prime}$ & $c^{\prime}$ & $h^{\prime}$ \\
26 & 4 & 13 \\
\hline
\end{tabular}

Table 1: Geometry of the 3-flap system analysed in Section 4. Each flap resembles the geometry of the Oyster 800 WEC designed by Aquamarine Power.

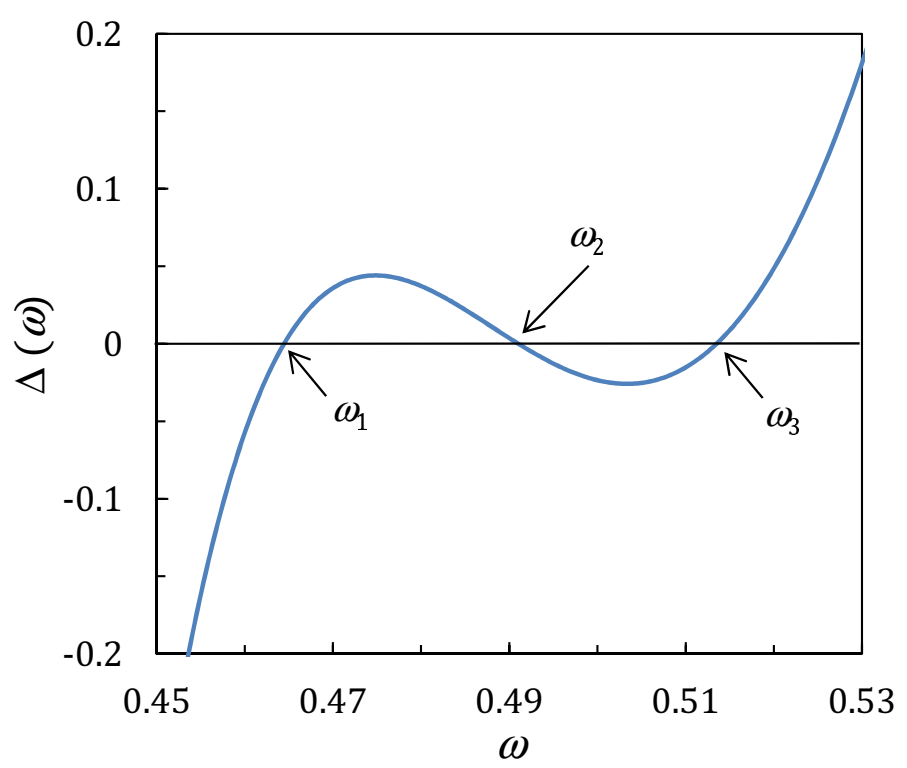

Figure 2: Solutions to Eq. (11) for the three-flap system of table 1.

and dimensions of the Oyster 800 WEC developed by Aquamarine Power. The values of the moment of inertia $I^{\prime}$ and the buoyancy torque $C^{\prime}$ are obtained by private communication with Aquamarine Power. As an order of magnitude, $I^{\prime}=O\left(10^{6}\right) \mathrm{kg} \mathrm{m}^{2}$ and $C^{\prime}=O\left(10^{7}\right) \mathrm{Nm}$.

\subsection{Natural modes}

Figure 2 shows a plot of the characteristic equation (11) with respect to the frequency $\omega$ for the system of table 1. Three roots of Eq. (11) are found in the positive real axis, which correspond to the three distinct natural frequencies of the system (eigenvalues). These are reported in table 2 together with the corresponding modal profiles (eigenvectors) of the oscillators. Those are the sought natural modes of the system and are sketched in figure 3 . Note that profile 2 is antisymmetric with respect to the centreline of the array, while profiles 1 and 3 are symmetric. Note also that the largest relative 


\begin{tabular}{cccccc}
\hline$i$ & $\omega_{i}$ & $T_{p}^{\prime}(\mathrm{s})$ & $\Theta_{1}\left(\omega_{i}\right)$ & $\Theta_{2}\left(\omega_{i}\right)$ & $\Theta_{3}\left(\omega_{i}\right)$ \\
1 & 0.4645 & 22.0270 & 1.00 & 1.39 & 1.00 \\
2 & 0.4910 & 20.8350 & 1.00 & 0.00 & -1.00 \\
3 & 0.5135 & 19.9235 & 1.00 & -1.40 & 1.00 \\
\hline
\end{tabular}

Table 2: Natural frequencies, periods (in physical variables) and modes of oscillations for the system of table 1 .

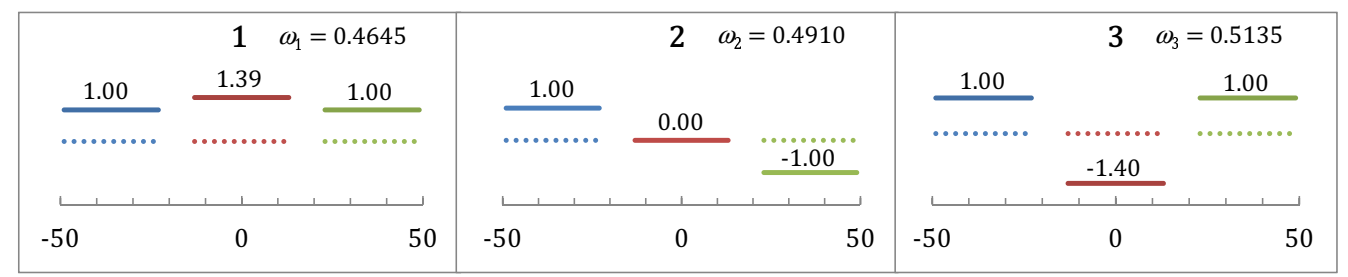

Figure 3: Modal profiles of the three-flap system of table 1 corresponding to the natural frequencies of table 2 (online version in colour).

displacement between two neighbouring flaps occurs with profile 3 , where neighbouring oscillators move in out-of-phase fashion.

\subsection{Wave power extraction}

Here we shall analyse the performance of the system of oscillators when they are used to extract energy from the waves. This corresponds to the analysis of the full damped forced system of Eq. (8). Figure 4 represents the behaviour of the absorption coefficient $Q_{M=3}(\omega)$ of Eq. (15) for the 3-flap system described in table 1, under normally incident waves. For this system, Eq. (15) simplifies to

$$
Q_{3}=\left(1+\frac{2 \nu_{11}+4 \nu_{12} \cos \left(\delta_{1}-\delta_{2}\right)\left|\frac{\Theta_{2}}{\Theta_{1}}\right|+2 \nu_{13}+\nu_{22}\left|\frac{\Theta_{2}}{\Theta_{1}}\right|^{2}}{\eta\left(2+\left|\frac{\Theta_{2}}{\Theta_{1}}\right|^{2}\right)},\right.
$$

which again depends on the relative amplitude of rotation $\left|\Theta_{2} / \Theta_{1}\right|$. The latter is not affected by exceedingly large resonant peaks (see again figure $3)$. The curve of figure 4 shows two minima for the absorption coefficient which are achieved close to the natural frequencies of the symmetric modes 1 and 3 depicted in figure 3 , respectively $\omega_{1}$ and $\omega_{3}$ of table 2 . When the 


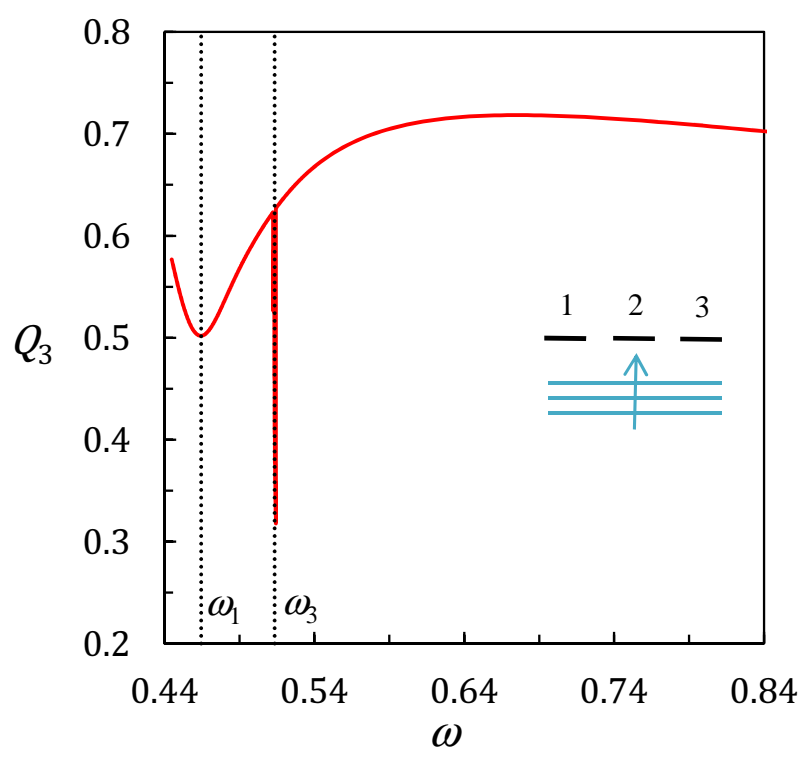

Figure 4: Absorption efficiency $Q_{M}(15)$ for a system of 3 in-line flap absorbers $(M=3)$ under normally incident waves. The vertical dotted lines show the natural frequencies of the system (see figure 2). Given the symmetry of the problem, only the symmetric natural modes corresponding to $\omega_{1}$ and $\omega_{3}$ (see figure 3 ) are excited at resonance (online version in colour). 
incident wave frequency is close to one of the resonant frequencies, the relevant motion-resonant mode is excited resulting in loss of absorption efficiency of the system. That is, the absorbers perform oscillations which not only increase the power capture $P$ but also largely enhance the power lost by radiation $W$, so that overall the absorption efficiency (15) drops. Note from figure 4 that the local minimum of $Q_{3}$ near $\omega_{1}$ is about $1 / 2$, i.e. it almost corresponds to the minimum absorption efficiency of a single flap tuned to body resonance (see Eq. 19). The absolute minimum of the $Q_{3}$ coefficient is reached near the third natural frequency $\omega_{3}$. There, strong outof-phase oscillations are triggered (see panel 3 of figure 3) which enhance the power lost by radiation, resulting in an inefficient functioning of the system. Producing more energy waste in order to generate more power is both a non-environmentally-sustainable cost and a non-optimal design principle. It is then clear that motion resonance is to be avoided in the design of a system of closely spaced, large flap-type absorbers. One viable alternative is to employ a larger number of much smaller devices, in which case trapped resonant modes can have a beneficial effect on the performance of the system (Sammarco et al., 2014).

\subsection{Influence of spacing on motion-resonant modes}

Indeed the spacing between the absorbers plays an important role in influencing the motion-resonant behaviour of the system and requires further investigation. Figure 5 shows several plots of the characteristic determinant $\Delta$ (11) versus the frequency $\omega$ for the three-flap system of table 1. Each curve refers to a specific value of the spacing $s$. The curve relevant to a single oscillator is also plotted, behaving almost as a straight line. The zero of this curve is clearly the natural frequency $\omega_{0}(12)$ of a single body due to buoyancy alone. Note from figure 5 that as $s$ increases, the zeroes of $\Delta$ become closer and the curve flattens near them, expectedly converging to the curve of the single oscillator. Now recall from Section 3 that the zeroes of $\Delta$ are the natural frequencies of the system. Hence the plots of figure 5 suggest a converging asymptotic behaviour of the array natural frequencies $\omega_{i}$ towards the natural frequency $\omega_{0}(12)$ at large spacing. Such behaviour is further confirmed by a quick asymptotic analysis of the characteristic equation (11) at large $s$. For $s>1$, it is expected that the mutual interactions between the oscillators become weak (Renzi et al., 2014a), so that the reflexive hydrodynamic terms become much larger than the mutual ones: $\left|\mu_{\alpha \alpha}\right| \gg\left|\mu_{\alpha \beta}\right|$. This is confirmed numerically by the plots of figure 6 , which show the behaviour 


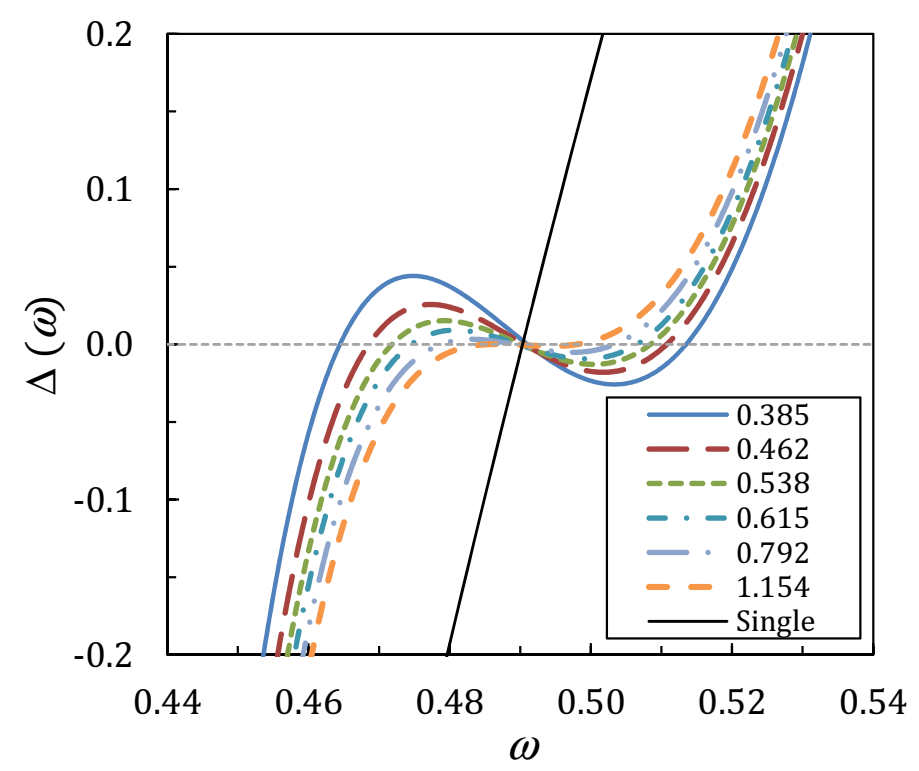

Figure 5: Plots of $\Delta$ (11) versus $\omega$ for the three-flap system of table 1.Various spacings are considered: $s=0.385\left(s^{\prime}=10 \mathrm{~m}\right), s=0.462\left(s^{\prime}=12 \mathrm{~m}\right), s=0.538\left(s^{\prime}=14 \mathrm{~m}\right), s=0.615$ $\left(s^{\prime}=16 \mathrm{~m}\right), s=0.792\left(s^{\prime}=20 \mathrm{~m}\right)$ and $s=1.154\left(s^{\prime}=30 \mathrm{~m}\right)$. The central black line shows the behaviour of $\Delta$ for a single flap. The zeroes of $\Delta$ identify the natural frequencies of each system (online version in colour). 

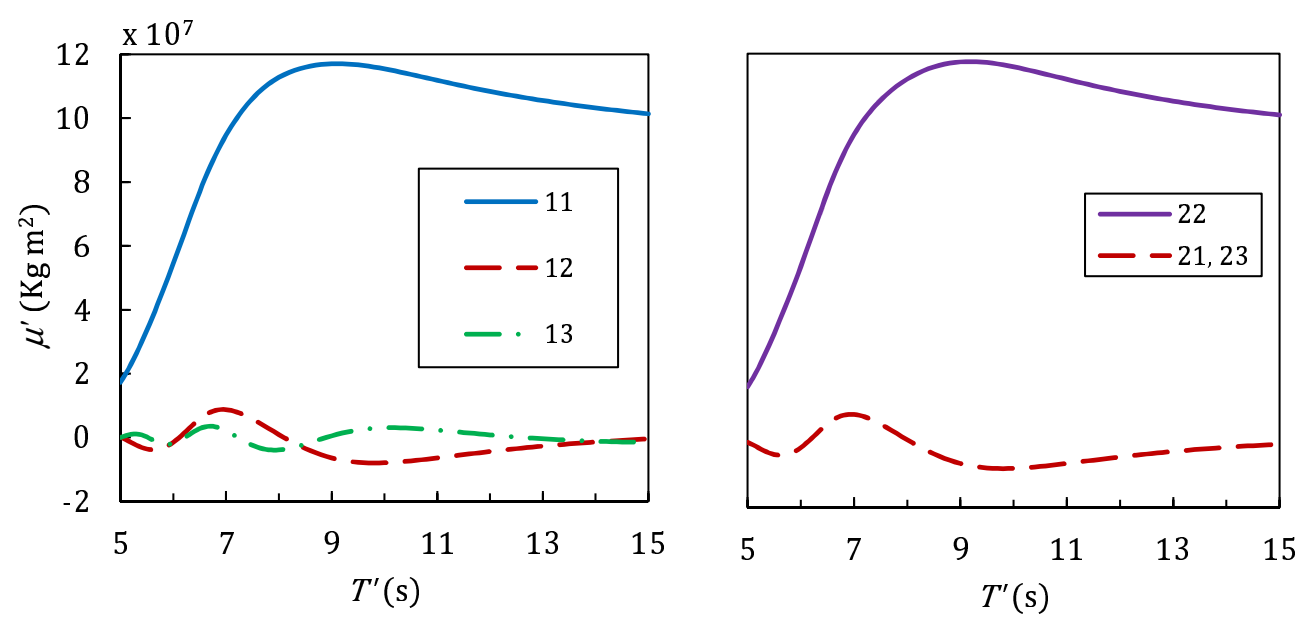

Figure 6: Plots of the added-inertia matrix components $\mu_{\alpha \beta}^{\prime}$ versus the period of oscillation in physical variables for a system of three flap absorbers with spacing $s^{\prime}=30 \mathrm{~m}$. The geometry of each flap is that of table 1 , so that $s=s^{\prime} / w^{\prime}=1.154$. The numerical calculations have been performed with the semi-analytical model of Renzi et al. (2014a). Note that $\left|\mu_{\alpha \alpha}^{\prime}\right| \gg\left|\mu_{\alpha \beta}^{\prime}\right|$ and $\mu_{11}^{\prime} \simeq \mu_{22}^{\prime}$ at all periods (online version in colour).

of the added inertia terms versus the period of oscillation for a system of largely spaced oscillators, in physical variables. As a consequence, at large $s$ the added-inertia matrix $\mathbf{M}$ can be approximated as a diagonal matrix and the characteristic equation (11) yields simply

$$
\omega_{i} \sim \sqrt{\frac{C}{I+\mu_{i i}}}, \quad i=1, \ldots, M .
$$

Furthermore, since the added inertia terms $\mu_{i i}$ naturally tend to equate the added inertia $\mu_{11}$ of an isolated oscillator as $s$ increases (see again figure 6, where $\mu_{11}^{\prime} \simeq \mu_{22}^{\prime}$ ), Eq. (20) finally yields

$$
\omega_{i} \sim \omega_{0}=\sqrt{\frac{C}{I+\mu_{11}}}, \quad i=1, \ldots, M, \quad s \rightarrow \infty .
$$

In conclusion, at large spacing the solutions of the characteristic equation (11) converge towards a single eigenvalue (21) of algebraic multiplicity $M$, which physically corresponds to the natural frequency of a single body (12). As a consequence of this dynamics, at large spacing the multi-resonant behaviour described in Section 3 is expected to be inhibited. The quantitative aspects of 

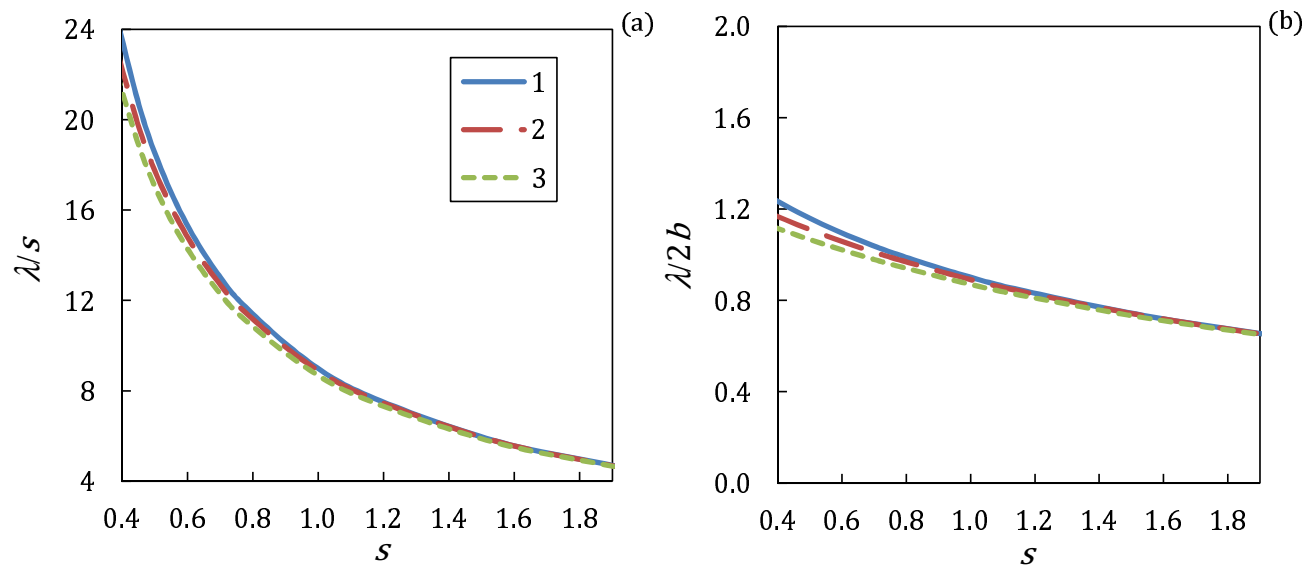

Figure 7: Plots of (a) $\lambda_{i} / s$ and (b) $\lambda_{i} /(2 b)$ versus the spacing $s$ for the three-flap system of table 1 . Solid lines: $i=1$; dashed lines: $i=2$; dotted lines $i=3$. In (b) the array full length is $b=2 s+3$. All the curves eventually converge for $s>1$.

this phenomenon can be further investigated by considering the ratio $\lambda_{i}^{\prime} / s^{\prime}=$ $\lambda_{i} / s$ between the resonant wavelengths $\lambda_{i}$ - each corresponding to the relevant natural frequency $\omega_{i}$ via the dispersion relation - and the spacing of the oscillators. Figure 7(a) shows the behaviour of $\lambda_{i} / s, i=1,2,3$, with respect to the spacing $s$ for the three-flap system under analysis. When $s<1$, the three curves can be distinguished neatly: the resonant wavelengths are different and the system exhibits the multi-resonant behaviour discussed in Section 3. As $s$ increases, the three curves merge into a single one, due to the convergence of the array resonant frequencies to the natural frequency of a single oscillator (see figure 5 and Eq. (21)): the system loses its multi-resonant characteristics. Figure 7(a) also shows that at small spacings the resonant wavelengths are much larger than the spacing itself, i.e. $\lambda_{i} / s \gg 1$. However, with respect to the total width of the array $b^{\prime}$, the resonant wavelengths roughly satisfy $\lambda_{i}^{\prime} /\left(2 b^{\prime}\right)=\lambda_{i} /(2 b) \simeq 1$, with $b=b^{\prime} / w^{\prime}$, as shown in figure $7(\mathrm{~b})$. This result can be explained by recurring to a simplified physical argument. Consider for example the radiation problem in which flap 1 (the first from the left) is oscillating at frequency $\omega$ and the other flaps are held fixed. A wave crest radiated by such a flap reaches the furthest flap (i.e. flap 3) roughly at time $t_{1} \simeq k b / \omega$. Then it is partially scattered back towards the first flap. The scattered wave crest will then reach flap 1 roughly at $t_{2} \simeq 2 k b / \omega$. If $t_{2}=T$, then the scattered wave will superimpose with a new crest radiated by the first flap, thus generating a strong resonant effect. Hence $T \simeq 2 k b / \omega$ 
is the sought resonant condition, which then yields $\lambda /(2 b) \simeq 1$. However, when the spacing $s$ is large, a single wave crest is not able anymore to travel twice the distance $b$ in one single period and the body-to-body resonant effect is inhibited.

\section{Conclusions}

In this paper, we investigated a resonant phenomenon involving large articulated damped oscillators under incoming waves in the open ocean, which gives rise to multiple motion resonances in the low-frequency range. Two requisites appear to be essential to excite such mechanism: (i) a close proximity of the oscillators (i.e. $s<1$ ) and (ii) low frequencies of the incident waves $(\omega<1)$. We showed that motion-resonant modes are responsible for strong out-of-phase oscillations of the bodies. For the first time, we also demonstrated that excitation of body-to-body resonances for a system of large articulated damped oscillators in waves is detrimental to the efficiency of wave energy absorption processes. Resonance produces more energy waste in order to produce more power. That is both a non-environmentally-sustainable cost for a renewable energy system. Our results, even in the limit of the linear potential flow theory, provide a new insight on the dynamics of large articulated damped oscillators, challenging the diffused belief that resonance is always beneficial to the performance of flap-type converters.

This publication has emanated from research conducted with the financial support of Science Foundation Ireland under Grant Number SFI/10/IN.1 /I2996. Fruitful discussion with Professor P. Sammarco and Dr G. Bellotti are kindly acknowledged. We acknowledge the useful comments of two anonymous referees.

\section{Appendix A. Computational aspects}

Numerical evaluation of the system of equations of motion (8) needs to be performed in order to determine the dynamic response of a system of $M$ oscillators. The added-inertia matrix $\mathbf{M}(\omega)$ and the radiation damping matrix $\mathbf{N}(\omega)$ in Eq. (8) are determined for each frequency $\omega$ by integrating numerically the radiation potentials $\phi_{R}^{(m)}$ over the surface of the oscillators, while the exciting torque vector $\mathbf{F}$ is found by integrating the diffraction potential $\phi_{D}$. Convergence tests for non-resonant frequencies were already carried out 
successfully in Renzi et al. (2014a), where the results were also compared satisfactorily with numerical data. In this paper, further convergence tests were performed to validate the model near resonant frequencies. Numerical integration was performed with a parallelised Mathematica ${ }^{\circledR}$ code on a highspeed computer equipped with an i7 $3.40 \mathrm{GHz}$ CPU and 16 GB RAM. An adaptive algorithm was used for the numerical evaluation of the integrals. The algorithm routinely subdivides the integration region until the error estimate achieves either the relative or the absolute tolerance required. In this paper, the relative error has been set to default $\left(10^{-7}\right)$ and the absolute error to $10^{-8}$. The maximum number of recursive subdivisions was set to 12 and was always sufficient to achieve the desired precision without any convergence warnings. Five vertical eigenmodes and eight Chebyshev polynomials - i.e. a $(5,8)$ configuration - were used. Convergence tests were undertaken for the 3-flap system of table 1 to ensure the reliability of the numerical calculations. Such layout was the most demanding in computational terms among the layouts analysed in this paper. Results obtained in the reference configuration $(5,8)$ were compared against those obtained with a $(5,10)$ and a $(6,8)$ configuration, respectively, and the relative errors were calculated. The system was more sensitive to an increase in the number of Chebyshev polynomials rather than of the vertical eigenmodes. Concerning the calculation of the hydrodynamic terms $\mathbf{F}, \mathbf{M}$ and $\mathbf{N}$, the largest relative error occurred near the resonant frequency $\omega_{3}$ and was $O\left(10^{-5}\right)$ for the added inertia $\mu_{11}$. Concerning the amplitude vector $\boldsymbol{\Theta}$, solution of Eq. (8), the largest relative error occurred again near the resonant frequency $\omega_{3}$ and was $O\left(10^{-3}\right)$ for the angle $\Theta_{2}$. Hence the $(5,8)$ configuration was safely adopted for all the numerical calculations performed in this paper. Coherence tests were also undertaken based on the reciprocity relations $\mu_{\alpha \beta}=\mu_{\beta \alpha}$ and $\nu_{\alpha \beta}=\nu_{\beta \alpha}$. The latter expressions were all satisfied in the $(5,8)$ configuration with a maximum relative error $O\left(10^{-10}\right)$.

\section{References}

Adamo, A., Mei, C.C., 2005. Linear response of Venice storm gates to incident waves. Proceedings of the Royal Society A 461, 1711-1734.

Babarit, A., 2010. Impact of long separating distances on the energy production of two interacting wave energy converters. Ocean Engineering 37, 718-729. 
Bouscasse, B., Colagrossi, A., Marrone, S., Antuono, M., 2013. Nonlinear water wave interaction with floating bodies in SPH. Journal of Fluids and Structures 42, 112-129.

Budal, K., 1977. Theory for absorption of wave power by a system of interacting bodies. Journal of Ship Research 21, 248-253.

Cruz, J. (Ed.), 2008. Ocean Wave Energy. Springer.

Falnes, J., 1980. Radiation impedance matrix and optimum power absorption for interacting oscillators in surface waves. Applied Ocean Research 2, 7580 .

Falnes, J., 2002. Ocean Waves and Oscillating Systems. Cambridge University Press, Cambridge.

Farhat, M., Enoch, S., Guenneau, S., Movchan, A.B., 2010. Broadband cylindrical acoustic cloak for linear surface waves in a fluid. Physical Review Letters 101, 134501.

Folley, M., Whittaker, T., Henry, A., 2007. The effect of water depth on the performance of a small surging wave energy converter,. Ocean Engineering 34, 1265-1274.

Gallagher, S., Tiron, R., Dias, F., 2013. A detailed investigation of the nearshore wave climate and the nearshore wave energy resource on the west coast of ireland, in: Proceedings of the 32nd International Conference on Ocean, Offshore and Arctic Engineering, OMAE2013, Nantes, France.

Heathershaw, A.D., 1982. Seabed-wave resonance and sandbar growth. Nature 296, 343-345.

Hu, X., Chan, C.T., 2005. Refraction of water waves by periodic cylinder arrays. Physical Review Letters 95, 154501.

Hu, X., Chan, C.T., Ho, K.M., Zi, J., 2011. Negative effective gravity in water waves by periodic resonator arrays. Physical Review Letters 106, 174501.

Kreyszig, E., 2006. Advanced Engineering Mathematics. John Wiley and Sons, Singapore. 
Liu, X., Lin, P., Shao, S., 2014. An ISPH simulation of coupled structure interaction with free surface flows. Journal of Fluids and Structures 48, $46-61$.

Maniar, H.D., Newman, J.N., 1997. Wave diffraction by a long array of cylinders. Journal of Fluid Mechanics 339, 309-330.

Mei, C., Sammarco, P., Chan, E.S., Procaccini, C., 1994. Subharmonic resonance of proposed storm gates for Venice Lagoon. Proceedings of the Royal Society A 444, 257-265.

Mei, C.C., Hara, T., Naciri, M., 1988. Note on Bragg scattering of water waves by parallel bars on the sea bed. Journal of Fluid Mechanics 186, $147-162$.

Mei, C.C., Stiassnie, M., Yue, D.K.P., 2005. Theory and Appliations of Ocean Surface Waves. World Scientific, Singapore.

Porter, R., Evans, D.V., 1999. Rayleigh-Bloch surface waves along periodic gratings and their connection with trapped modes in waveguides. Journal of Fluid Mechanics 386, 233-258.

Rafiee, A., Dias, F., 2013. Numerical simulation of wave impact on an oscillating wave surge converter, in: Proceedings of the ASME 2013 32nd International Conference on Ocean, Offshore and Arctic Engineering OMAE2013, Nantes, France.

Renzi, E., Abdolali, A., Bellotti, G., Dias, F., 2014a. Wave-power absorption from a finite array of oscillating wave surge converters. Renewable Energy $63,55-68$.

Renzi, E., Dias, F., 2012. Resonant behaviour of an oscillating wave energy converter in a channel. Journal of Fluid Mechanics 701, 482-510.

Renzi, E., Dias, F., 2013a. Hydrodynamics of the Oscillating Wave Surge Converter in the open ocean. European Journal of Mechanics B/Fluids 41, $1-10$.

Renzi, E., Dias, F., 2013b. Relations for a periodic array of flap-type wave energy converters. Applied Ocean Research 39, 31-39. 
Renzi, E., Doherty, K., Henry, A., Dias, F., 2014b. How does Oyster work? The simple interpretation of Oyster mathematics. European Journal of Mechanics B/Fluids. http://dx.doi.org/10.1016/j.euromechflu.2014.03.007.

Renzi, E., Sammarco, P., 2010. Landslide tsunamis propagating around a conical island. Journal of Fluid Mechanics 650, 251-285.

Ruan, Z., Fan, S., 2010. Superscattering of light from subwavelength nanostructures. Physical Review Letters 105, 013901.

Sammarco, P., Michele, S., d'Errico, M., 2014. Flap gates farm: from Venice lagoon defense to resonating wave energy production. Part 1. Natural modes. Applied Ocean Research 43, 206-213.

Sammarco, P., Renzi, E., 2008. Landslide tsunamis propagating along a plane beach. Journal of Fluid Mechanics 598, 107-119.

Sammarco, P., Tran, H.H., Gottlieb, O., Mei, C.C., 1997a. Subharmonic resonance of Venice gates in waves. Part 2. Sinusoidally modulated incident waves. Journal of Fluid Mechanics 349, 327-359.

Sammarco, P., Tran, H.H., Mei, C.C., 1997b. Subharmonic resonance of Venice gates in waves. Part 1. Evolution equation and uniform incident waves. Journal of Fluid Mechanics 349, 295-325.

Sarkar, D., Renzi, E., Dias, F., 2013. Wave power extraction by an oscillating wave surge converter in random seas, in: Proceedings of the ASME 2013 32nd International Conference on Ocean, Offshore and Arctic Engineering OMAE2013, Nantes, France.

Sarkar, D., Renzi, E., Dias, F., 2014. Modelling wave farms of oscillating wave surge converters. Proceedings of the Royal Society of London A 20140118.

Stefanakis, T.S., Dias, F., Dutykh, D., 2011. Local run-up amplification by resonant wave interaction. Physical Review Letters 107.

Thomas, G.P., Evans, D.V., 1981. Arrays of three-dimensional wave-energy absorbers. Journal of Fluid Mechanics 108, 67-88. 
Wei, Y., Rafiee, A., Dias, F., 2013a. On the viscous effects in the interaction of water waves with an oscillating wave surge converter, in: Proceedings of the 10th European Wave and Tidal Energy Conference, EWTEC13, Aalborg, Denmark.

Wei, Y., Rafiee, A., Elsaesser, B., Dias, F., 2013b. Numerical simulation of an oscillating wave surge converter, in: Proceedings of the ASME 2013 32nd International Conference on Ocean, Offshore and Arctic Engineering OMAE2013, Nantes, France.

Whittaker, T., Folley, M., 2012. Nearshore oscillating wave surge converters and the development of Oyster. Philosophical Transaction of the Royal Society A 370, 345-364. 